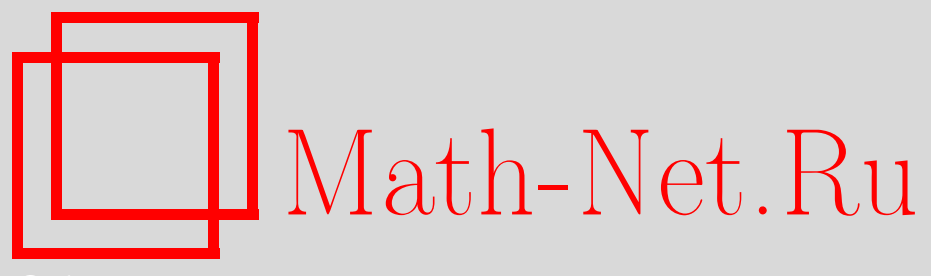

С. Д. Глызин, А. Ю. Колесов, Н. Х. Розов, Явление буферности в одном двумерном кусочно-линейном отображении из радиофизики, Матем. заметки, 2007, том 81, выпуск 4, 507-514

DOI: https://doi.org/10.4213/mzm3693

Использование Общероссийского математического портала Math-Net.Ru подразумевает, что вы прочитали и согласны с пользовательским соглашением http: //www . mathnet.ru/rus/agreement

Параметры загрузки:

IP: 54.196 .121 .252

26 апреля 2023 г., 12:42:28

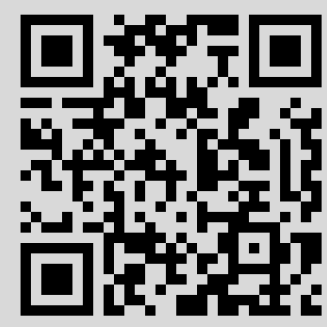


Том 81 выпуск 4 апрель 2007

УДК 517.926

\section{Явление буферности в одном двумерном кусочно-линейном отображении из радиофизики}

\section{С. Д. Глызин, А. Ю. Колесов, Н. Х. Розов}

Изучается вопрос об аттракторах двумерного отображения

$$
(u, v) \rightarrow(v,-(1-\mu) u-F(v)), \quad F(v)=\left\{\begin{array}{cc}
q_{1} & \text { при } v>0, \\
0 & \text { при } v=0, \\
-q_{2} & \text { при } v<0,
\end{array}\right.
$$

где $0<\mu \ll 1, q_{1}, q_{2}>0$. Данное отображение служит математической моделью автогенератора с релейным усилителем и с отрезком длинной линии без искажений в цепи обратной связи. Устанавливается, что при надлежащем уменьшении параметра $\mu$ в рассматриваемой системе сосуществуют устойчивые циклы сколь угодно больших периодов. Показывается также, что при $\mu \rightarrow 0$ общее число этих циклов неограниченно растет, т.е. реализуется явление буферности.

Библиография: 4 названия.

1. Рассмотрим автогенератор, блок-схема которого изображена на рис. 1. Предполагаем, что вольт-амперная характеристика его нелинейного элемента имеет вид, представленный на рис. 2. Считаем, далее, что в цепь обратной связи этого генератора включен отрезок $0 \leqslant x \leqslant 1$ длинной линии с равномерно

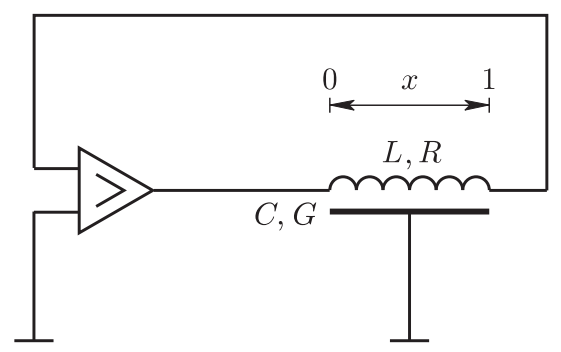

Рис. 1

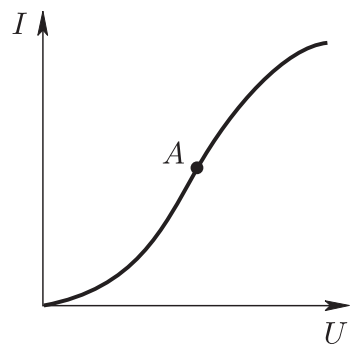

Рис. 2

распределенными индуктивностью $L$, емкостью $C$, активным сопротивлением $R$ и проводимостью $G$. Тогда, как показано в [1], математической моделью описанного

Работа выполнена при поддержке Российского фонда фундаментальных исследований (грант № 05-01-01004).

(C) С. Д. Глызин, А. Ю. Колесов, Н. Х. Розов, 2007 
генератора служит следующая краевая задача, рассматриваемая на отрезке $0 \leqslant$ $x \leqslant 1$ :

$$
\begin{gathered}
U_{t}=-V_{x}-\alpha_{1} U, \quad V_{t}=-U_{x}-\alpha_{2} V, \\
\left.V\right|_{x=1}=\left.\beta_{1} U\right|_{x=1},\left.\quad \beta_{2} V\right|_{x=0}+\left.U\right|_{x=0}+\varphi\left(\left.K U\right|_{x=1}\right)=0 .
\end{gathered}
$$

Здесь $U, V$ - нормированные переменные составляющие напряжения и силы тока в линии;

$$
\alpha_{1}=G \sqrt{\frac{L}{C}}, \quad \alpha_{2}=R \sqrt{\frac{C}{L}}, \quad \beta_{1}=\frac{1}{R_{\mathrm{Bx}}} \sqrt{\frac{L}{C}}, \quad \beta_{2}=R_{\mathrm{Bыx}} \sqrt{\frac{C}{L}},
$$

где $R_{\mathrm{Bx}}, R_{\text {вых }}$ - входное и выходное сопротивления усилителя; $K>0$ - коэффициент усиления, а функция $\varphi(z) \in C^{\infty}(\mathbb{R})$ получается из нелинейной характеристики усилителя в результате сдвига рабочей точки $A$ (см. рис. 2) в начало координат. В связи с этим считаем, что $\varphi^{\prime}(z)>0$ при всех $z \in \mathbb{R}, \varphi(0)=0$ и, что самое главное,

$$
\begin{gathered}
\varphi(z) \rightarrow q_{1} \quad \text { при } z \rightarrow+\infty, \quad \varphi(z) \rightarrow-q_{2} \quad \text { при } z \rightarrow-\infty, \\
z \varphi^{\prime}(z) \rightarrow 0 \quad \text { при } z \rightarrow \pm \infty,
\end{gathered}
$$

где $q_{1}, q_{2}>0$.

Исследование аттракторов краевой задачи (1) проводилось в ряде работ (см., например, [1]-[3]). Было установлено, что при согласованном уменьшении параметров (2) и увеличении коэффициента усиления $K$ в этой системе происходит неограниченное накапливание устойчивых циклов, т.е. наблюдается явление буферности. Следует отметить, впрочем, что в упомянутых работах была изучена лишь локальная динамика задачи (1) в малой окрестности ее нулевого состояния равновесия. Вопрос же о том, что происходит в ней при существенном увеличении $K$, когда заведомо перестает работать какая-либо локальная теория, до сих пор оставался открытым. В настоящей статье ответ на этот вопрос удается получить в случае линии без искажений, т.е. при дополнительном предположении

$$
\alpha_{1}=\alpha_{2}=\alpha>0 \text {. }
$$

Анализ автоколебательных процессов в рассматриваемой модели базируется на следующем специальном приеме. Проинтегрируем фигурирующую в (1) линейную систему методом Римана. В результате убеждаемся, что при выполнении равенств (4) любое решение указанной системы, удовлетворяющее линейному граничному условию в точке $x=1$, имеет вид

$$
\begin{aligned}
& U(t, x)=\exp (-\alpha t)\left[h(t-(x-1))+\frac{1-\beta_{1}}{1+\beta_{1}} h(t+(x-1))\right], \\
& V(t, x)=\exp (-\alpha t)\left[h(t-(x-1))-\frac{1-\beta_{1}}{1+\beta_{1}} h(t+(x-1))\right],
\end{aligned}
$$

где $h(t)$ - произвольная непрерывная функция (пока речь идет об обобщенных решениях класса $C$, хотя в дальнейшем мы расширим этот класс). Подставляя, далее, формулы (5) во второе граничное условие из (1) и полагая $\theta(t)=\exp (-\alpha t) h(t)$, $\eta(t)=\theta(t-1)$, приходим к системе разностных уравнений, которая в векторной форме записи имеет вид

$$
z(t+1)=H(z(t)),
$$


где

$$
\begin{gathered}
z=(\eta, \theta)^{T}, \quad H(z)=\left(\theta,-\varkappa_{1} \eta-\varkappa_{2} \varphi(2 K \theta)\right)^{T}, \\
\varkappa_{1}=\frac{1-\beta_{1}}{1+\beta_{1}} \cdot \frac{1-\beta_{2}}{1+\beta_{2}} \exp (-2 \alpha), \quad \varkappa_{2}=\frac{\exp (-\alpha)}{\left(1+\beta_{2}\right)} .
\end{gathered}
$$

Теория разностных уравнений с непрерывным временем развита в монографии [4]. Напомним, что произвольному начальному условию $z_{0}(t) \in C_{0}$, где $C_{0}-$ нелинейное многообразие непрерывных при $-1 \leqslant t \leqslant 0$ вектор-функций $z_{0}(t)=\left(\eta_{0}(t), \theta_{0}(t)\right)^{T}$, удовлетворяющих условиям согласования

$$
\eta_{0}(0)=\theta_{0}(-1), \quad \theta_{0}(0)=-\varkappa_{1} \eta_{0}(-1)-\varkappa_{2} \varphi\left(2 K \theta_{0}(-1)\right),
$$

соответствует единственная непрерывная при всех $t \geqslant-1$ вектор-функция $z(t)$ : $z(t)=z_{0}(t)$ при $-1 \leqslant t \leqslant 0$, обращающая уравнение (6) в тождество. Добавим еще, что эта функция строится методом шагов.

Итак, на многообразии $C_{0}$ определена сильно непрерывная по $t$ нелинейная полугруппа

$$
T\left[t, z_{0}(s)\right]=z(t+s), \quad-1 \leqslant s \leqslant 0, \quad t \geqslant 0,
$$

аттракторы которой при $t \rightarrow \infty$, собственно говоря, и представляют для нас интерес. Следует, однако, заметить (см. [4]), что в наиболее содержательном случае, когда соответствующее разностному уравнению (6) дискретное отображение в $\mathbb{R}^{2}$

$$
z \rightarrow H(z)
$$

имеет аттрактор, отличный от неподвижной точки, почти все траектории полугруппы (8) при $t \rightarrow \infty$ становятся асимптотически разрывными. А это, в свою очередь, означает, что в описанной ситуации данная полугруппа вообе не имеет аттракторов в пространстве $C_{0}$.

Из всего вышесказанного вытекает, что необходимо каким-либо образом расширить фазовое пространство разностного уравнения (6), включив в него разрывные функции. Один из возможных способов сделать это заключается в следующем. Фиксируем некоторое разбиение отрезка $[-1,0]$ точками $-1=\tau_{0}<\tau_{1}<\cdots<\tau_{n}=0$, $n \geqslant 1$ и рассмотрим банахово пространство

$$
E\left(\tau_{0}, \tau_{1}, \ldots, \tau_{n}\right)=C\left(\left[\tau_{0}, \tau_{1}\right] ; \mathbb{R}^{2}\right) \times C\left(\left[\tau_{1}, \tau_{2}\right] ; \mathbb{R}^{2}\right) \times \cdots \times C\left(\left[\tau_{n-1}, \tau_{n}\right] ; \mathbb{R}^{2}\right)
$$

Определим затем в пространстве (10) оператор $\widetilde{H}$ по формуле

$$
\left(z_{1}(t), z_{2}(t), \ldots, z_{n}(t)\right) \stackrel{\widetilde{H}}{\rightarrow}\left(H\left(z_{1}(t)\right), H\left(z_{2}(t)\right), \ldots, H\left(z_{n}(t)\right)\right) .
$$

Далее, фиксируем произвольно начальное условие

$$
\widetilde{z}_{0}(t)=\left(z_{1,0}(t), z_{2,0}(t), \ldots, z_{n, 0}(t)\right) \in E\left(\tau_{0}, \tau_{1}, \ldots, \tau_{n}\right)
$$

и рассмотрим положительную полутраекторию

$$
\left(z_{1, m}(t), z_{2, m}(t), \ldots, z_{n, m}(t)\right)=\widetilde{H}^{m}\left(\widetilde{z}_{0}(t)\right), \quad m \geqslant 0
$$

отображения (11) с этим начальным условием. 
На следующем этапе установим соответствие между траекториями (13) и некоторыми решениями разностного уравнения (6). А именно, положим

$$
z(t)=z_{k, m}(t-m) \quad \text { при } t \in\left[m+\tau_{k-1}, m+\tau_{k}\right), \quad k=1, \ldots, n, \quad m \geqslant 0 .
$$

Получившуюся в итоге функцию назовем разрывным решением уравнения (6), а построенные с учетом формул (14), (5) разрывные функции $U(t, x), V(t, x), t \geqslant 0$, $x \in[0,1]$, по определению будем считать разрывными решениями исходной краевой задачи (1). Более того, любую определенную таким образом разрывную траекторию уравнения (6) или исходной системы (1) назовем устойчивой (неустойчивой) по Ляпунову, если аналогичным свойством обладает соответствующая траектория дискретного отображения (11) в пространстве (10).

Из приведенных определений ясно, что если, например, отображение (11) имеет экспоненциально устойчивый цикл, то разрывный цикл с теми же свойствами устойчивости существует и у исходной краевой задачи (1). Для отыскания же у этого отображения требуемых циклов заметим следующее. Рассмотрим вспомогательное отображение (9) в $\mathbb{R}^{2}$ и предположим, что оно имеет экспоненциально устойчивый цикл периода $p \geqslant 1$ с компонентами $z_{k}^{*}, k=1, \ldots, p$. Тогда, как нетрудно увидеть, экспоненциально устойчивые циклы (некоторые из которых могут совпадать) исходного отображения (11) порождаются любыми начальными условиями (12), в которых $z_{k, 0}(t) \equiv c_{k}, k=1, \ldots, n$, а векторы $c_{k} \in \mathbb{R}^{2}$ независимо друг от друга принимают значения из множества $\left\{z_{k}^{*}\right\}$.

Подводя итог, отметим, что проблема отыскания устойчивых разрывных циклов краевой задачи (1) свелась к нахождению устойчивых циклов отображения (9), которое после нормировок и переобозначений

$$
u=\frac{\eta}{\varkappa_{2}}, \quad v=\frac{\theta}{\varkappa_{2}}, \quad 2 K \varkappa_{2} \rightarrow K, \quad \mu=1-\varkappa_{1}
$$

преобразуется к виду

$$
(u, v) \rightarrow(v,-(1-\mu) u-\varphi(K v)) .
$$

Исследование отображения (16) будем проводить при дополнительных условиях $K=\infty, 0<\mu \ll 1$. Первое из них означает, что в качестве нелинейного элемента генератора взято идеальное реле, а второе заведомо выполняется при традиционных в данной тематике предположениях о малости параметров $\alpha, \beta_{1}, \beta_{2}$ (см. (2), (7), (15)). Далее, перейдем в системе (16) к пределу при $K \rightarrow \infty$ и воспользуемся свойствами (3). В итоге после нормировок $2 u / q_{2} \rightarrow u, 2 v / q_{2} \rightarrow v$ получим отображение

$$
\Pi(\mu):(u, v) \rightarrow(v,-(1-\mu) u-\psi(v))
$$

с релейной нелинейностью

$$
\psi(v)=\left\{\begin{array}{rl}
2 d & \text { при } v>0, \\
0 & \text { при } v=0, \\
-2 & \text { при } v<0,
\end{array} \quad d=\frac{q_{1}}{q_{2}},\right.
$$

которое и является объектом дальнейшего анализа. 
2. Приступим к описанию основной конструкции. С этой целью фиксируем произвольно натуральное $l$ и введем в рассмотрение на плоскости $(u, v)$ прямоугольник $\Omega_{l}$ с вершинами

$$
\begin{array}{ll}
A_{l}=(-d,-d+(l+1)(d+1)), & B_{l}=(-d+(l+1)(d+1),-d), \\
C_{l}=(1,-d-l(d+1)), & D_{l}=(-d-l(d+1), 1) .
\end{array}
$$

Рассмотрим затем все точки вида $(-d+k(d+1),-d+m(d+1)), k, m \in \mathbb{Z}$, лежащие на границе $\Gamma_{l}$ этого прямоугольника. Занумеруем их, начиная с вершины $A_{l}$, в порядке обхода контура $\Gamma_{l}$ по часовой стрелке и обозначим получившиеся точки через $P_{k}$, $k=0,1, \ldots, 4 l+1$. В результате имеем набор точек

$$
S_{l}=\left\{P_{k}, k=0,1, \ldots, 4 l+1\right\},
$$

расположение которых в простейшем случае $l=1, d=1$ показано на рис. 3. Далее, нам потребуется отображение

$$
\Pi_{0}:(u, v) \rightarrow(v,-u-\psi(v)),
$$

получающееся из (17) при $\mu=0$. Нетрудно убедиться, что отображение (19) не только оставляет инвариантным множество (18), но и справедливо следующее более сильное утверждение.

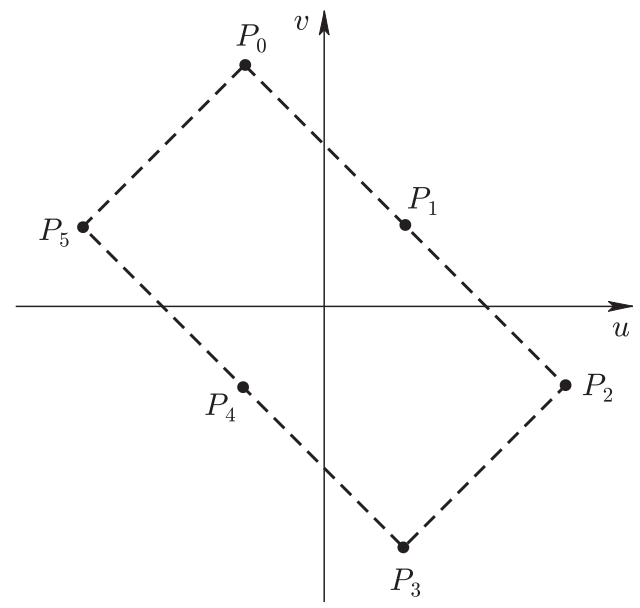

Рис. 3

Теорема 1. Имеет место цепочка равенств

$$
\Pi_{0}\left(P_{k}\right)=P_{k+l+1}, \quad k=0,1, \ldots, 4 l+1,
$$

где, естественно, предполагается, что $P_{n}=P_{m}$ в случае $n=m(\bmod 4 l+2)$.

Доказательство. Рассмотрим элементы множества (18), лежащие на ребрах $D_{l} A_{l}$ и $A_{l} B_{l}$. Таковыми являются, очевидно, точки с координатами

$$
\begin{aligned}
(-d-(k-1)(d+1),-d+(l-k+2)(d+1)), & k=1,2, \ldots, l+1, \\
(-d+m(d+1),-d+(l-m+1)(d+1)), & m=1,2, \ldots, l+1,
\end{aligned}
$$


соответственно. Непосредственная проверка показывает, что каждая точка первого семейства с номером $k$ под действием отображения (19) переходит в точку второго семейства с номером $m=l-k+2$. Ясно также, что, двигаясь вдоль контура $\Gamma_{l}$ в направлении по часовой стрелке от произвольной точки множества (21) к ее образу, мы "перепрыгиваем" ровно через $l$ промежуточных точек. А это эквивалентно выполнению соотношений (20) для элементов множества $S_{l}$, принадлежащих ребpy $D_{l} A_{l}$.

На следующем этапе обратимся к точкам множества $S_{l}$, находящимся на ребре $B_{l} C_{l}$, координаты которых имеют вид

$$
(-d+r(d+1),-d-(l-r+1)(d+1)), \quad r=1,2, \ldots, l+1 .
$$

Заметим, далее, что образом любой точки (22) с номером $m, m \leqslant l$, является точка $(23)$ с номером $r=l-m+1$. А отсюда, как и в предыдущем случае, вытекает справедливость для семейства $(22)$ при $m \leqslant l$ требуемых равенств $(20)$.

Итак, при любых значениях параметра $d>0$ соотношения (20) выполняются для всех элементов множества (18), лежащих в полуплоскости $v>0$. Проверять же их справедливость для оставшихся элементов из $S_{l}$ нет необходимости, поскольку как само отображение (19), так и множество (18) инвариантны относительно преобразования $u / d \rightarrow-u, v / d \rightarrow-v$ и последующей замены $1 / d \rightarrow d$. Теорема доказана.

В связи с установленной теоремой естественно возникает вопрос о характере поведения траекторий отображения (19) с начальными условиями из множества (18). Ответ на него содержится в следующем утверждении.

ТеОрема 2. В случае четного l точки (18) образуют единый цикл $G_{l}$ отображения (19) периода $4 l+2$, мультипликаторы которого равны -1 . Если же l нечетно, то множество $S_{l}$ распадается на два иикла $G_{l}^{(1)}, G_{l}^{(2)}$ периода $2 l+1$, каждый из которых имеет комплексные мультипликаторы $\pm i$.

ДокАзАтЕльство. Существование циклов, лежащих в множестве (18), очевидно, так как это множество инвариантно относительно отображения (19) и состоит из конечного числа элементов. Далее, пусть $P_{k_{0}} \in S_{l}$ - начальное условие какого-либо из этих циклов, а $s$ - наименьшее натуральное число такое, что $\Pi_{0}^{s}\left(P_{k_{0}}\right)=P_{k_{0}}$. Из геометрических соображений ясно (см. (20)), что прежде, чем вернуться в первоначальное положение, точка $P_{k_{0}}$ совершает некоторое количество полных "обходов" вдоль контура $\Gamma_{l}$. А это значит, что

$$
(l+1) s=m(4 l+2)
$$

при некотором натуральном $m$. Заметим также, что поскольку нас интересует наименьший период $s$, то в равенстве $(24)$ в качестве $m$ следует взять наименьшее натуральное число, для которого выражение $4 m-2 m /(l+1)$ получается целым. А отсюда, автоматически следует, что $m /(l+1)=1 / 2$ или $m /(l+1)=1$.

Первая из отмеченных альтернатив реализуется при нечетном $l$ и в этом случае все циклы из множества (18) имеют периоды $s=2 l+1$. Тем самым, таких циклов в точности два, а их мультипликаторы, являющиеся собственными значениями матрицы

равны $\pm i$.

$$
\left(\begin{array}{rr}
0 & 1 \\
-1 & 0
\end{array}\right)^{s}
$$


В случае четного $l$ с необходимостью имеем $m=l+1$, а значит, в множестве $S_{l}$ существует единственный цикл периода $s=4 l+2$. Снова привлекая матрицу (25), убеждаемся, что мультипликаторы этого цикла равны -1 . Теорема доказана.

Обратимся теперь к исходному отображению (17). Из теорем 1, 2 вытекает, что при $\mu \rightarrow 0$ количество его устойчивых циклов неограниченно растет. Точнее говоря, справедлив следующий результат.

Теорема 3. По любому натуральному $m_{0}$ можно указать такое достаточно малое $\mu_{0}=\mu_{0}\left(m_{0}\right)>0$, что при всех $0<\mu \leqslant \mu_{0}$ отображение (17) имеет аналитически зависящие от н экспоненциально устойчивые ицклы

$$
G_{2 m-1}^{(1)}(\mu), \quad G_{2 m-1}^{(2)}(\mu), \quad G_{2 m}(\mu), \quad m=1,2, \ldots, m_{0},
$$

обращающиеся при $\mu=0$ в иикль

$$
G_{2 m-1}^{(1)}, \quad G_{2 m-1}^{(2)}, \quad G_{2 m}, \quad m=1,2, \ldots, m_{0},
$$

системы (19).

ДоКАЗАтЕЛЬСтво. Для построения циклов (26) с требуемыми свойствами рассмотрим серию уравнений

$$
\Pi^{s}(\mu)(P)=P,
$$

где $s=4 m-1$ или $s=8 m+2$, а $m=1,2, \ldots, m_{0}$. Заметим, далее, что поскольку мультипликаторы циклов (27) равны $\pm i$ или -1 , то к любому из уравнений (28) применима теорема о неявной функции по переменной $P$ в точке $\mu=0, P=P_{*}$, где $P_{*}$ - произвольный элемент множества $S_{2 m-1}$ (в случае $s=4 m-1$ ) или $S_{2 m}$ (при $s=8 m+2)$. Таким образом, все эти уравнения имеют аналитические по $\mu$ решения, что и означает существование интересующих нас циклов (26).

Перейдем теперь к вопросу об устойчивости найденных циклов. Нетрудно увидеть, что их мультипликаторы определяются из матриц

$$
\left(\begin{array}{cc}
0 & 1 \\
\mu-1 & 0
\end{array}\right)^{s}
$$

где $s=4 m-1$ или $s=8 m+2$ соответственно. Остается добавить, что во всех случаях собственные значения $\lambda_{1}, \lambda_{2}$ этих матриц таковы, что $\left|\lambda_{1}\right|=\left|\lambda_{2}\right|=(1-\mu)^{s / 2}<1$. Теорема 3 доказана.

3. Сделаем некоторые выводы о динамике исходной краевой задачи (1), вытекающие из проделанного анализа. В связи с этим обратим внимание, что в силу наложенных на функцию $\varphi(z)$ ограничений (см. (3)) предельное равенство

$$
\lim \frac{2}{q_{2}} \varphi(K v)=\psi(v), \quad K \rightarrow \infty,
$$

справедливо в метрике $C^{1}(\Omega)$, где $\Omega$ - произвольное замкнутое множество, не содержащее точку $v=0$. А отсюда, в свою очередь, следует, что устойчивые циклы, аналогичные циклам (26), сохраняются при любом фиксированном достаточно малом $\mu>0$ и в рамках системы (16), если, конечно, параметр $K$ в ней надлежащим образом велик. 
Итак, отображение (16) имеет устойчивые циклы, причем количество последних за счет выбора параметров $\mu, K$ может быть сделано сколь угодно большим. Вспоминая, далее, о связи между решениями систем (16) и (1) (см. п. 1), убеждаемся, что феномен буферности наблюдается и в рамках исходной краевой задачи (1).

В заключение добавим, что, как следует из содержащихся в [4] результатов, при отходе от условия (4), т.е. при $\alpha_{1}=\alpha, \alpha_{2}=\alpha+\nu, \alpha=$ const $>0,|\nu| \ll 1$, сглаживания разрывных колебаний не происходит: аналоги построенных выше разрывных циклов "выживают" и лишь немного деформируются.

\section{СПИСОК ЦИТИРОВАННОЙ ЛИТЕРАТУРЫ}

[1] А. Ю. Колесов, Е.Ф. Мищенко, Н. Х. Розов, “Асимптотические методы исследования периодических решений нелинейных гиперболических уравнений”, Тр. МИАН, 222 (1998), 1-191.

[2] А. Ю. Колесов, Н. Х. Розов, "Автоколебания в RCLG-линии с малыми искажениями”, Дифферени. уравнения, 34:11 (1998), 1559-1561.

[3] А. Ю. Колесов, Н. Х. Розов, "Явление буферности в RCLG-автогенераторе: теоретический анализ и результаты эксперимента”, Тр. МИАН, 233 (2001), 153-207.

[4] А. Н. Шарковский, Ю. Л. Майстренко, Е. Ю. Романенко, Разностные уравнения и их приложения, Наукова думка, Киев, 1986.

\section{С. Д. Глызин}

Ярославский государственный университет им. П. Г. Демидова

\section{А. Ю. Колесов}

Ярославский государственный университет им. П. Г. Демидова

E-mail: kolesov@uniar.ac.ru

\section{Н. Х. Розов}

Московский государственный университет

им. М. В. Ломоносова
Поступило

30.03.2005

Исправленный вариант

09.02 .2006 